\title{
Abdominal Ultrasound and its diagnostic accuracy in diagnosing Acute Appendicitis: A Meta-Analysis
}

\author{
Jian $\mathrm{Fu}^{1}$, Xu Zhou ${ }^{1}$, Liang $\mathrm{Chen}^{1}$, and Sheng $\mathrm{Lu}^{1}$ \\ ${ }^{1}$ Nantong University Affiliated Haian Hospital
}

May 22, 2021

\begin{abstract}
Background: Acute appendicitis (AA) is a common cause for abdominal pain encountering unnecessary surgeries in emergency departments. The present meta-analysis aims to assess the accuracy of abdominal ultrasound in suspected acute appendicitis cases in terms of sensitivity, specificity, and post-test odds for positive and negative results. Materials and Methods: An extensive and systematic search was conducted in Medline (via PubMed), Cinahl (via Ebsco), Scopus, and Web of Sciences from 2010 till the end of March 2021. Two authors analyzed studies for inclusion, collected results, and conducted analyses separately. The histopathological study on tissue collected during appendectomy served as the gold standard for determining the final diagnosis. The accuracy was determined by evaluating sensitivity, specificity, positive predictive value (PPV), negative predictive value (NPV), diagnostic odds ratio. Results: Out of 3193 references, a total of 18 studies were selected. Overall sensitivity of $77.2 \%$ (95\% CI $-75.4-78.9 \%)$ and specificity of $60 \%$ (95\% CI - 58\% - 62\%) were observed. The diagnostic odds ratio of $6.88(95 \%$ CI $1.99-23.82)$ was obtained. Conclusion: Abdominal ultrasound shows significant accuracy of diagnosis in patients with suspected acute appendicitis.
\end{abstract}

\section{Abdominal Ultrasound and its diagnostic accuracy in diagnosing Acute Appendicitis: A Meta- Analysis}

\section{Abstract}

Background: Acute appendicitis (AA) is a common cause for abdominal pain encountering unnecessary surgeries in emergency departments. The present meta-analysis aims to assess the accuracy of abdominal ultrasound in suspected acute appendicitis cases in terms of sensitivity, specificity, and post-test odds for positive and negative results.

Materials and Methods: An extensive and systematic search was conducted in Medline (via PubMed), Cinahl (via Ebsco), Scopus, and Web of Sciences from 2010 till the end of March 2021. Two authors analyzed studies for inclusion, collected results, and conducted analyses separately. The histopathological study on tissue collected during appendectomy served as the gold standard for determining the final diagnosis. The accuracy was determined by evaluating sensitivity, specificity, positive predictive value (PPV), negative predictive value (NPV), diagnostic odds ratio.

Results: Out of 3193 references, a total of 18 studies were selected. Overall sensitivity of $77.2 \%$ (95\% CI $-75.4-78.9 \%)$ and specificity of $60 \%$ (95\% CI - 58\% - 62\%) were observed. The diagnostic odds ratio of $6.88(95 \%$ CI $1.99-23.82)$ was obtained.

Conclusion: Abdominal ultrasound shows significant accuracy of diagnosis in patients with suspected acute appendicitis.

Keywords: Acute Appendicitis; Ultrasound; Computed Tomography (CT scan); Histopathology; Emergency Department. 


\section{Review criteria: how did you gather, select and analyze the information you considered in}

your review?

An extensive and systematic search was conducted in Medline (via PubMed), Cinahl (via Ebsco), Scopus, and Web of Sciences from 2010 till the end of March 2021. Two authors analyzed studies for inclusion, collected results, and conducted analyses separately. The histopathological study on tissue collected during appendectomy served as the gold standard for determining the final diagnosis.

\section{Message for the clinic: what is the 'take-home' message for the clinician?}

Although imaging with CT has significantly lowered the negative appendectomy rates but still due to its skilled operator use, high cost, high ionization radiation exposure risks, and its complexity for interpretation makes ultrasound technique an efficient diagnostic aid mainly in suspected cases of children, young females, and elderly patients

\section{Introduction}

Acute appendicitis (AA) is considered one of the most common causes of surgical emergencies worldwide. ${ }^{1}$ The reported mortality rate is from $<1 \%$ in younger patients up to $5 \%$ in the elderly ${ }^{2,3}$. Abdominal pain is one of the most common cause of acute appendicitis, yet $34 \%$

of cases ${ }^{4,5}$ are still misdiagnosed, which results in unnecessary surgery. This high rate of negative appendectomy can be decreased by careful and accurate diagnosis of appendicitis, thus preventing acute appendicitis from progressing to perforation and peritonitis. ${ }^{6}$

Abdominal ultrasound (US), computed tomography (CT), and magnetic resonance imaging (MRI) have also been used in the identification or exclusion of AA. Sensitivity and specificity for the US in identifying AA have been reported to range from 71 to 92 percent and 83 percent, respectively, for normal contrast-enhanced CT 98 and 91 percent, and MRI 97 and 93 percent $^{7-9}$.

Computed Tomography (CT) is the most preferred diagnostic imaging modality to rule out AA in the adult population. Although its accuracy is high, with sensitivities ranging from $90-96 \%$ and specificities ranging from 94-98\%; however, there are certain limitations, including radiation exposure, risk of contrast administration, increased resource utilization, high $\operatorname{cost}^{7,8}$, and development of future malignancies ${ }^{9}$. To eliminate such limitations, the incidence of negative appendicectomy rate and perforation, clinicians often go for imaging modalities such as abdominal ultrasound (US) as an alternative diagnostic approach as it is easy, inexpensive method, easily portable, and has high precision ${ }^{10}$ in cases of suspected appendicitis both in children and adults.

CT or US did not improve the diagnostic precision of AA. Despite its confirmed low diagnostic accuracy, the US has been listed as a potential method for diagnosing AA because it does not require radiation. However, despite being a non-ionizing process, the question remains whether the US can contribute to the management of patients with AA suspicion without causing further management delays. Patients with stomach pain who do not have AA are exposed to invasive surgery if the condition is misdiagnosed. It can happen in up to $34 \%$ of $\operatorname{cases}^{4,5}$.

Rationale: When patients with AA are misdiagnosed as not having the condition, a mandatory appendectomy may be postponed, and severe complications may occur, with a mortality rate of about 1.5 percent $^{2}$. Legal charges against both nonsurgical and surgical subspecialties have been identified in cases of delayed or incorrect diagnosis leading to adverse effects. As a result, it is essential to correctly identify AA in patients who exhibit symptoms and signs suggestive of the condition.

Objective: The present study is an approach to rule out the diagnostic accuracy of abdominal ultrasound compared to the gold standard, i.e., histopathology in cases of acute appendicitis (AA) in terms of sensitivity and specificity for positive and negative US results.

\section{Materials and Methods}


We followed the Standards for Reporting of Diagnostic Accuracy Studies (STARD) normative recommendations in this study with the registration number NU\#/IRB/2020/1022.

\section{Search Strategy}

The present meta-analysis is an extensive search conducted in Medline (via PubMed), Cinahl (via Ebsco), Scopus, Web of Sciences, from 2010 till the end of March 2021. The search was performed based on the keywords related to diagnostic accuracy, abdominal ultrasound, acute appendicitis, diagnosis, decreased CT use, and ultrasonography. All articles selected were based on STARD guidelines. The selection was irrespective of language or publication status or whether the study conducted was done prospectively or retrospectively. Table.1summarizes the demographic details of the studies included from the search query of the Medline database with the considered variables. The primary focus of the present study was to assess the efficacy of ultrasound for the cases of acute appendicitis in all age groups. To rule out the efficacy of ultrasound in cases of acute appendicitis; sensitivity, specificity, positive predictive value (PPV), negative predictive value (npv), and diagnostic odds ratio's were assessed with the help of true positive (TP), false positive $(\mathrm{FP})$, true negative $(\mathrm{TN})$, false negative $(\mathrm{FN})$ values.

It did not matter whether the data was compiled prospectively or retrospectively or what language it was written in. Two authors (JF and XZ) separately scanned the sources for related studies. For publications that at least one of the writers thought was significant, full texts of the sources were collected. To further exclude obsolete references, complete texts were obtained. Abstracts were only used if they included enough information for the study. Two researchers (LC and SL) independently collected data from included research.

\section{Inclusion and Exclusion criteria}

The studies evaluated the diagnostic accuracy of ultrasound for acute appendicitis in all age groups from 2010 to 2021 and where the histopathology report for the same was defined as the reference standard were included in the present study. Only full-text data were included in the present study. Exclusion criteria included insufficient data, reference standard other than histopathology report, any relevant studies but published before 2010, and pregnant females suffering from acute appendicitis.

Evaluation of the analytical standard

The quality assessment of diagnostic accuracy tests assessment tool (QUADAS-2) ${ }^{36}$ was used to determine the methodological quality of the included studies. The methodological validity of the included studies was evaluated by two reviewers (JF and XZ) separately. SL was in charge of resolving any disagreements between authors.

\section{Statistical Analysis}

A 2 X 2 table was made, based on which pooled sensitivity, specificity, and diagnostic odds ratio were calculated employing the DerSimonian Lair technique. The diagnostic odds ratio was also evaluated with a higher DOR value indicating better diagnostic accuracy of the test. The Cochran $\mathrm{Q}$ statistic and $\mathrm{I}^{2}$ index evaluated the heterogeneity of the studies included. Meta disc software was used for the creation of forest plots. We also presented the data obtained from the various studies in the form of summary points of sensitivity and specificity in receiver operating characteristics (SROC) space with corresponding $95 \%$ confidence regions created using Review Manager $5 .^{11}$

\section{Analysis of Sensitivity}

Excluding participants with ambiguous findings can lead to an overestimation of diagnostic test accuracy. As a result, we conducted a sensitivity study in which we incorporated uninterpretable results in the analysis and evaluated diagnostic precision in which all uninterpretable results were considered incorrect, comparing the outcomes to those of the principal analysis, which removed uninterpretable results.

Investigation of sources of heterogeneity 
We used metaregression to investigate heterogeneity in the included experiments, introducing various sources of heterogeneity as covariates and fitting a bivariate model. To see whether a covariate has a significant impact on the description sensitivity and precision, we used the probability ratio test. For any of the subgroups, a p-value of 0.05 was found statistically significant. Full-text publication versus abstracts, high versus low risk of bias in included studies, prospective versus retrospective studies, studies that included only adults versus those that included mixed adult and paediatric populations, proportion of female participants, proportion of obese patients, type of ultrasound probe, and ultrasonographer experience were among the sources of heterogeneity that we investigated.

\section{Results}

\section{Literature Search Results}

Through electronic scans, we found a total of 3193 studies. By reading titles and abstracts, we were able to exclude 973 on reading titles and abstracts and 2035 invalid references. Out of 185 studies, around 131 studies were excluded based on duplicity. Full-text publications were required for final screening was 54 out of which, 36 were excluded based on the inclusion criteria. The study and meta-analysis contained 18 studies that met the inclusion criteria, i.e., based on the accuracy of abdominal ultrasound for acute cases of appendicitis, as shown in Figure 1. Inappropriate comparison criteria and inadequate evidence to create $2 \times 2$ tables for review were the key reasons for the omission.

Table 1 shows the demographic details of the studies included in the present meta-analysis describing study author, year of publishing, study type, study duration, total sample size, age, gender, details of sonographer, type of US probe used, sample size in which ultrasound was conducted, histopathology report which was considered as gold standard for the comparison and any other method of correlation used for diagnosis. A total of 4209 patients were included in the present meta-analysis. All studies were released as full-text papers, six of which were prospective, eight of which were retrospective, and four of which were cross-sectional. The participants' average age ranged from 14 to 60 years old, and the majority of research did provide information about the operator's background (13 out of 18) or the kind of US probe used (12 out of 18).

Risk of Bias Assessment

Individual reports' estimated sensitivity ranged from 75.4 to 78.9 percent, and specificity from 58 to 62 percent. Thus, according to the QUADAS-2 tool, all of the included experiments had a low chance of bias (Table 2).

\section{Meta-analysis Results}

The overall sensitivity of the abdominal ultrasound scan in acute appendicitis was $77.2 \%$ (95 \% CI - 75.4$78.9 \%$ ), as shown in Figure 2. The overall specificity was $60 \%$ (CI-58\%-62\%), as shown in Figure 3. The overall positive likelihood ratio (PPV) was 2.62 (95\%CI-1.57-4.35), as shown in Figure 4. and the overall negative likelihood ratio (NPV) was 0.45 (95\%CI-0.28-0.74) as shown in Figure 5. Figure 6 demonstrated an SROC plot showing an estimate of sensitivity versus specificity and area under the SROC curve. The diagnostic odds ratio was 6.88(1.99-23.82) at $95 \% \mathrm{CI}$, thus demonstrating greater accuracy of abdominal ultrasound in diagnosing acute appendicitis, as shown in Figure 7.

The proportion of female participants, number of prospective studies, female participants, type of ultrasound probe, Ultrasonographer experience, and clinical probability of acute appendicitis were the covariates that showed statistically significant effects on summary outcomes in the subgroup study (Table 3).

\section{Discussion}

Definitive diagnosis in acute appendicitis has always been challenging because of its non-specific symptoms, signs, and laboratory findings which usually can mimic several other pathologies ${ }^{29}$. It is considered to be one of the most common abdominal emergency surgery. However, to avoid such emergency surgeries, Computed tomography $(\mathrm{CT})$ scan is considered as the gold standard in diagnosing acute appendicitis patients, and it is seen in the past that preoperative imaging with CT has significantly lowered the negative appendectomy 
rates (NARs) to $1.7 \% 30,31$, but it exposes to ionizing radiation, is expensive and time-consuming and has its diagnostic insufficiencies. ${ }^{32}$

The present Meta-analysis was an effort to rule out the efficacy of abdominal ultrasound in diagnosing suspected cases of acute appendicitis in all age groups as it can be misdiagnosed, especially in young women, children, and elderly patients. This Meta-analysis was a systematic update from 2010 to 2021, and a total of 18 articles were selected to rule out the sensitivity, specificity, PPV, NPV, and Diagnostic odd ratios. The present analysis showed an overall sensitivity of $77 \%$ with $95 \%$ CI varying from $75 \%$ to $79 \%$ based on the studies included. The studies included a wide range of sensitivity varying from $50 \%$ to $100 \%$ (95\% CI $-41 \%-100 \%)$. The present analysis showed an overall specificity of $60 \%$ with $95 \%$ CI varying from $58 \%$ to $62 \%$. The studies included showed a wide range of sensitivity varying from $0 \%$ to $97 \%$ (95 \% CI - $0 \%-$ $98 \%$ ); when compared to other previous studies.

Similarly, Doria et al. ${ }^{32}$ compared CT and ultrasound in pediatric and adult populations. Again, surgery or follow-up was the gold standard. In the adult population, the combined sensitivity and specificity were 83 and 93 percent, respectively.

Giljaca V et al. ${ }^{33}$ showed a sensitivity of $69 \%$ and specificity of $81 \%$, which was different from the present study. The present study showed a high sensitivity rate compared to Giljaca et al. ${ }^{33}$, stating the ability to identify acute appendicitis patients more accurately. Another similar Meta-analysis was performed by Orr et al. ${ }^{34}$, showing sensitivity and specificity of $84.7 \% \& 92.1 \%$; however, the specificity of Orr et al. was very high when compared with the present meta-analysis showing a high ability to identify the patients without acute appendicitis, which differ from the present analysis. Orr et al. concluded that the US should not diagnose acute appendicitis cases, whereas the present study differs from Orr et al., showing accuracy for the US.

Another study done by Weston et al. 35 showed high sensitivity and specificity with a value of $88.3 \%$ \& $92.3 \%$, but this study did not take any reference standards when compared to the present study we took histopathology as the gold standard to compare the results of US to reduce the chances of false-negative rate. The positive likelihood ratio and negative likelihood ratio were 2.62 with a $95 \%$ CI of $1.57-4.35$ and 0.45 (95 $\%$ CI $-0.28 \%-0.74 \%)$. The diagnostic odds ratio of the present study was 6.88 (95\% CI $1.99-23.82$ ), showing a reasonable accuracy rate of abdominal ultrasound in diagnosing acute appendicitis. The SROC curve obtained in the present analysis shows the combined effect of sensitivity and specificity, indicating the inclination of the curve towards the top left depicting good diagnostic accuracy of abdominal ultrasound.

Likewise, only Korean papers were reviewed by Yu et al. ${ }^{37}$. Although most of the included participants were checked up on, surgery and histopathology do not seem to be the reference norm. The US had a sensitivity and specificity of 86.7 and 90.0 percent, respectively.

Van Randen et al. specifically compared CT and US; however, surgery was not the reference norm in all patients, and others were followed up without surgery. The US had a sensitivity and accuracy of 78 and 83 percent, respectively. Carroll et al. compared the sensitivity and specificity of the US performed by surgeons to histopathology or US performed by a radiologist, with sensitivity and specificity of 92 and 96 percent, respectively.

Only the histopathology record of the surgical specimen served as the reference standard in our research. As a result, our study's sensitivity and accuracy are much more minor than previously reported. This disparity may result from a rigidly enforced standard under which all patients were subjected to surgery. In addition, this fact may lead to an underestimation of sensitivity in our sample because the patient group was more chronically ill.

The limitation of the present study is that the variability in the type of sonographer as skilled and experienced radiologists can reduce the chance of false-negative results. The diagnostic accuracy of the US could be compared with other methods of imaging to see the variability. Analysis of studies showing accuracy based on techniques using Color Doppler to ultrasound examination and various scoring systems based on patient's 
history, physical examination, and laboratory tests can further improve the diagnostic accuracy rate of ultrasound in acute appendicitis.

\section{Conclusion}

Although imaging with CT has significantly lowered the negative appendectomy rates but still due to its skilled operator use, high cost, high ionization radiation exposure risks, and its complexity for interpretation makes ultrasound technique an efficient diagnostic aid mainly in suspected cases of children, young females, and elderly patients. In addition, it is a simple, non-invasive, non-ionizing radiation technique, and its easy availability makes it an effective diagnostic alternative to reduce the rate of unnecessary surgeries in acute appendicitis.

Acknowledgment: None Declared.

Statement of Ethics: All procedures performed in the study were in accordance with the institutional and/or national research committee's standards and with the 1964 Helsinki declaration and its later amendments or comparable ethical standards.

Conflict of Interest Statement: The authors declare that they have no competing interests.

Funding Sources: No Funding was received.

Author Contributions: JF: Concept and designed the study; XZ: analyzed data and drafting of the manuscript; LC: Collected the data and helped in data analysis; SL: Proofreading and final editing and guarantor of the manuscript.

"All authors read and approved the final version of the manuscript."

\section{References}

1. Douglas CD, Macpherson NE, Davidson PM, Gani JS. Randomised controlled trial of ultrasonography in diagnosis of acute appendicitis, incorporating the Alvarado score. BMJ. 2000 Oct 14;321(7266):91922 .

2. Flum DR, Morris A, Koepsell T, Dellinger EP. Has misdiagnosis of appendicitis decreased over time? A population-based analysis. JAMA. 2001 Oct 10;286(14):1748-53.

3. Lee SL, Walsh AJ, Ho HS. Computed tomography and ultrasonography do not improve and may delay the diagnosis and treatment of acute appendicitis. Arch Surg.2001;136:556-562.

4. Wilms IM, Suykerbuyk-de Hoog DE, de Visser DC, Janzing HM. Appendectomy versus antibiotic treatment for acute appendicitis. Cochrane Database Syst Rev. 2020 Oct 1;10:CD008359.

5. Bergeron E. Clinical judgment remains of great value in the diagnosis of acute appendicitis. Can J Surg. 2006 Apr;49(2):96-100.

6. Lane MJ, Liu DM, Huynh MD, Jeffrey RB Jr., Mindelzun RE, Katz DS. Suspected acute appendicitis: nonenhanced helical CT in 300 consecutive patients. Radiology . 1999; 213(2):341-6.

7. Gwynn LK. The diagnosis of acute appendicitis: clinical assessment versus computed tomography evaluation. J Emerg Med. 2001;21: 119-23.

8. Raman SS, Lu DSK, Kadell BM, Vodopich DJ, Sayre J, Cryer H. Accuracy of nonfocused helical CT for the diagnosis of acute appendicitis: a 5-year review. AJR Am J Roentgenol. 2002;178:1319-25.

9. Lessin MS, Chan M, Catallozzi M, et al. Selective use of ultrasonography for acute appendicitis in children. Am J Surg. 1999;177: 193-6.

10. Pedram A, Asadian F, Roshan N. Diagnostic Accuracy of Abdominal Ultrasonography in Pediatric Acute Appendicitis. Bull Emerg Trauma 2019;7(3):278-283.

11. Review Manager (RevMan) [Computer Program] (2012) Version 5.2. Copenhagen: The Nordic Cochrane Centre, The Cochrane Collaboration.

12. Samudra S S, Munde A S. Comparative study between clinical diagnosis using modified Alvarado score and ultrasound imaging in decreasing negative appendectomy rate. Int Surg J. 2021 Apr;8(4):11851189 . 
13. Austin-Page LR, Pham PK, Elkhunovich M. Evaluating Changes in Diagnostic Accuracy of Ultrasound for Appendicitis: Does Practice Make Perfect? J Emerg Med. 2020 Oct;59(4):563-572.

14. Farooq A, Zameer S, Khadim R. Diagnostic Accuracy Of Ultrasound In Acute Appendicitis In Comparison With Alvarado Score Keeping Histopathological Correlation As Gold Standard. Pak Armed Forces Med $J$ 2020; 70 (3): 80\%-11.

15. Crocker C, Akl M, Abdolell M, Kamali M, Costa AF. Ultrasound and CT in the Diagnosis of Appendicitis: Accuracy With Consideration of Indeterminate Examinations According to STARD Guidelines. AJR Am J Roentgenol. 2020 Sep;215(3):639-644.

16. Dhatt S, Sabhaney V, Bray H, Skarsgard E D. Improving the diagnostic accuracy of appendicitis using a multidisciplinary pathway. J Pediatr Surg . 2020;55(5):889-892.

17. Khan U, Kitar M, Krichen I, Maazoun K, Ali Althobaiti R, Khalif M, Adwani M. To determine validity of ultrasound in predicting acute appendicitis among children keeping histopathology as gold standard. Ann Med Surg (Lond). 2018 Dec 18;38:22-27.

18. Mirza WA, Naveed MZ, Khandwala K. Utility and Accuracy of Primary and Secondary Ultrasonographic Signs for Diagnosing Acute Appendicitis in Pediatric Patients. Cureus. 2018 Dec 27;10(12):e3779.

19. Tyler P D, Carey J, Stashko E, et al. The Potential Role Of Ultrasound In The Work-Up Of Appendicitis In The Emergency Department. J Emerg Med . 2019;56(2):191-196.

20. Shahbazipar M, Seyedhosseini J, Vahidi E, Motahar Vahedi HS, Jahanshir A. Accuracy of ultrasound exam performed by emergency medicine versus radiology residents in the diagnosis of acute appendicitis. Eur J Emerg Med. 2019 Aug;26(4):272-276.

21. Karimi E, Aminianfar M, Zarafshani K, Safaie A. The Accuracy of Emergency Physicians in Ultrasonographic Screening of Acute Appendicitis; a Cross Sectional Study. Emerg (Tehran). 2017;5(1):e22.

22. Hussain S, Rahman A, Abbasi T, Aziz T. Diagnostic Accuracy Of Ultrasonography In Acute Appendicitis. J Ayub Med Coll Abbottabad . 2014;26(1):12-17.

23. Parsijani P J, Zarandi NP, Paydar S, Abbasi H, Bolandparvaz S. Accuracy of Ultrasonography in Diagnosing Acute Appendicitis.Bull Emerg Trauma 2013;1(4):158-163.

24. Sezer T O, Gulece B, Zalluhoglu N, et al. Diagnostic Value of Ultrasonography in Appendicitis. Adv Clin Exp Med . 2012; 21(5): 633-636.

25. Burford JM, Dassinger MS, Smith SD. Surgeon-performed ultrasound as a diagnostic tool in appendicitis. J Pediatr Surg. 2011;46:1115-20.

26. Gökçe AH, Aren A, Gökçe FS, Dursun N, Barut AY. Akut apandisitte ultrasonografinin güvenilirliği [Reliability of ultrasonography for diagnosing acute appendicitis]. Ulus Travma Acil Cerrahi Derg. 2011 Jan;17(1):19-22. Turkish.

27. Peixoto R O; Nunes T A; Gomes C A, TCBC-MG. Indices of diagnostic abdominal ultrasonography in acute appendicitis. Influence of gender and physical constitution, time evolution of the disease and experience of radiologist. Rev. Col. Bras. Cir. 2011; 38(2): 105-111.

28. Memisoglu K, Karip B, Mestan M, Onur E. The value of preoperative diagnostic tests in acute appendicitis, retrospective analysis of 196 patients. World J Emerg Surg. 2010 Feb 11;5:5.

29. Becker T, Kharbanda A, Bachur R. Atypical clinical features of pediatric appendicitis. Acad Emerg Med. 2007;14(2):124-129.

30. Raja AS, Wright C, Sodickson AD, et al. Negative appendectomy rate in the era of CT: an 18-year perspective. Radiology.2010;256:460-465.

31. Soyer P, Dohan A, Eveno C, et al. Pitfalls and mimickers at 64-section helical CT that cause negative appendectomy: an analysis from 1057 appendectomies. Clin Imaging. 2013;37:895-901.

32. Doria AS, Moineddin R, Kellenberger CJ, et al. US or CT for diagnosis of appendicitis in children and adults? A meta-analysis. Radiology . 2006;241(1):83-94.

33. Giljaca V, Nadarevic T, Poropat G, Nadarevic VS, Stimac D. Diagnostic Accuracy of Abdominal Ultrasound for Diagnosis of Acute Appendicitis: Systematic Review and Meta-analysis. World J Surg. 2017 Mar;41(3):693-700.

34. Orr RK, Porter D, Hartman D. Ultrasonography to evaluate adults for appendicitis: decision making 
based on meta-analysis and probabilistic reasoning. Acad Emerg Med. 1995; 2:644-650.

35. Weston AR, Jackson TJ, Blamey S. Diagnosis of appendicitis in adults by ultrasonography or computed tomography: a systematic review and meta-analysis. Int $J$ Technol Assess Health Care . 2005; 21:368379 .

36. Whiting PF, Rutjes AWS, Westwood ME, et al. QUADAS-2: a revised tool for the quality assessment of diagnostic accuracy studies. Ann Intern Med. 2011;155:529-536.

37. Yu SH, Kim CB, Park JW, et al. Ultrasonography in the diagnosis of appendicitis: evaluation by meta-analysis. Korean J Radiol. 2005;6:267-277.

38. van Randen A, Bipat S, Zwinderman AH, et al. Acute appendicitis: meta-analysis of diagnostic performance of CT and graded compression US related to prevalence of disease. Radiology. 2008;249(1):97106.

39. Carroll PJ, Gibson D, El-Faedy O, et al. Surgeon-performed ultrasound at the bedside for the detection of appendicitis and gallstones: systematic review and meta-analysis. Am J Surg. 2013 Jan;205(1):1028.

\section{FIGURE LEGENDS}

1. Figure 1: Flow chart diagram for article inclusion based on STARD guidelines

2. Figure 2: Sensitivity of ultrasound in acute appendicitis cases

3. Figure 3: Specificity of ultrasound in acute appendicitis cases

4. Figure 4: Positive Likelihood ratio of ultrasound in acute appendicitis cases

5. Figure 5: Negative Likelihood ratio of ultrasound in acute appendicitis cases

6. Figure 6: SROC curve of ultrasound in acute appendicitis cases

7. Figure 7: Diagnostic OR of ultrasound in acute appendicitis cases

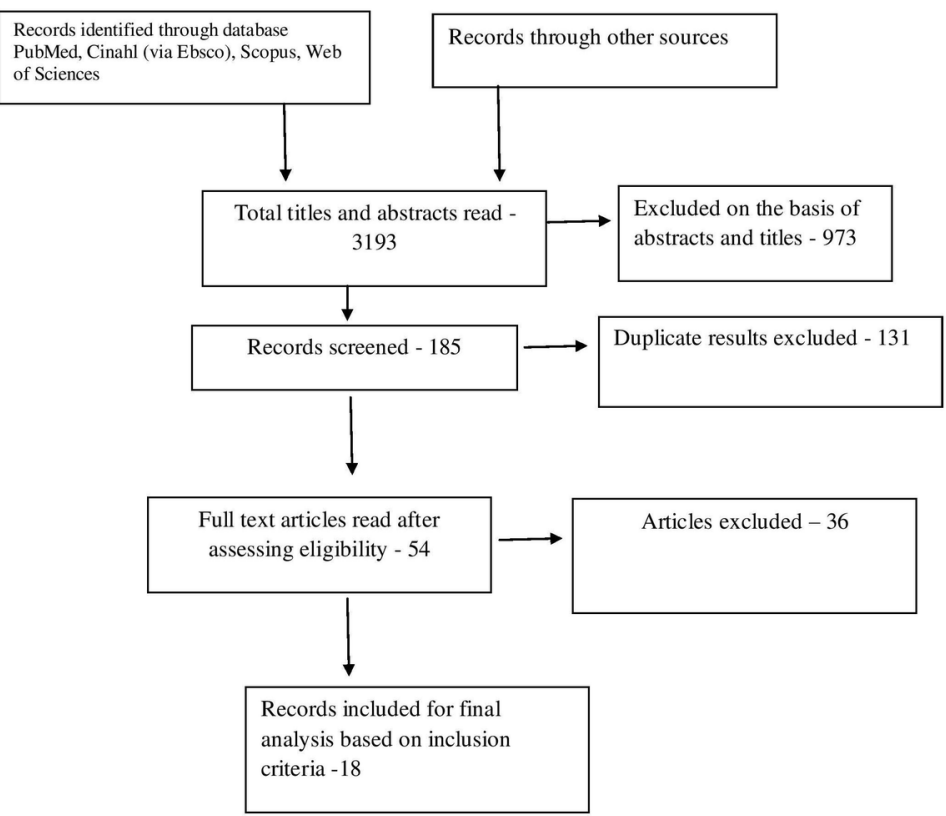


Figure 2: Sensitivity of ultrasound in acute appendicitis cases

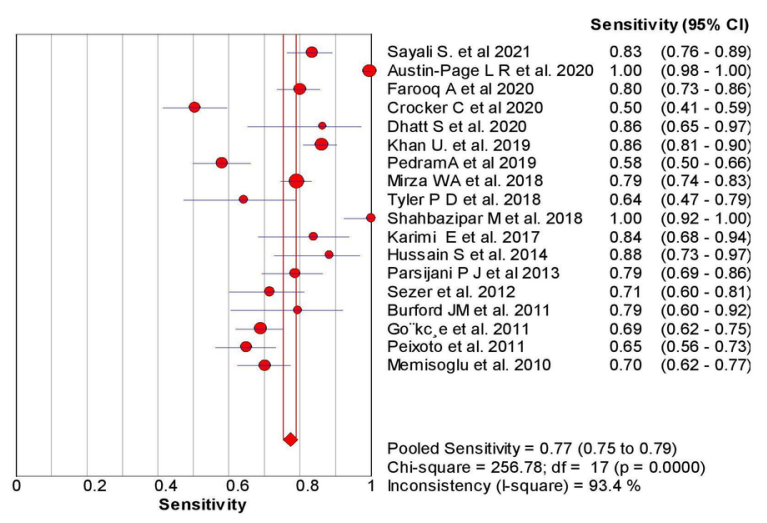

Figure 3: Specificity of ultrasound in acute appendicitis cases

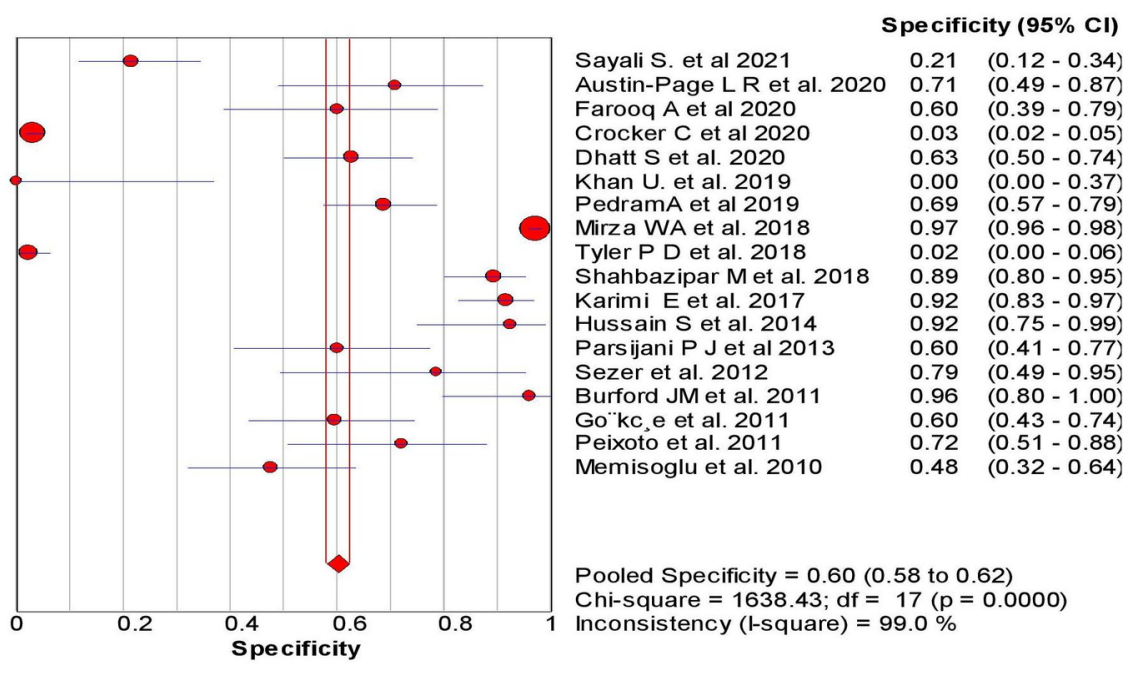


Figure 4: Positive Likelihood ratio of ultrasound in acute appendicitis cases

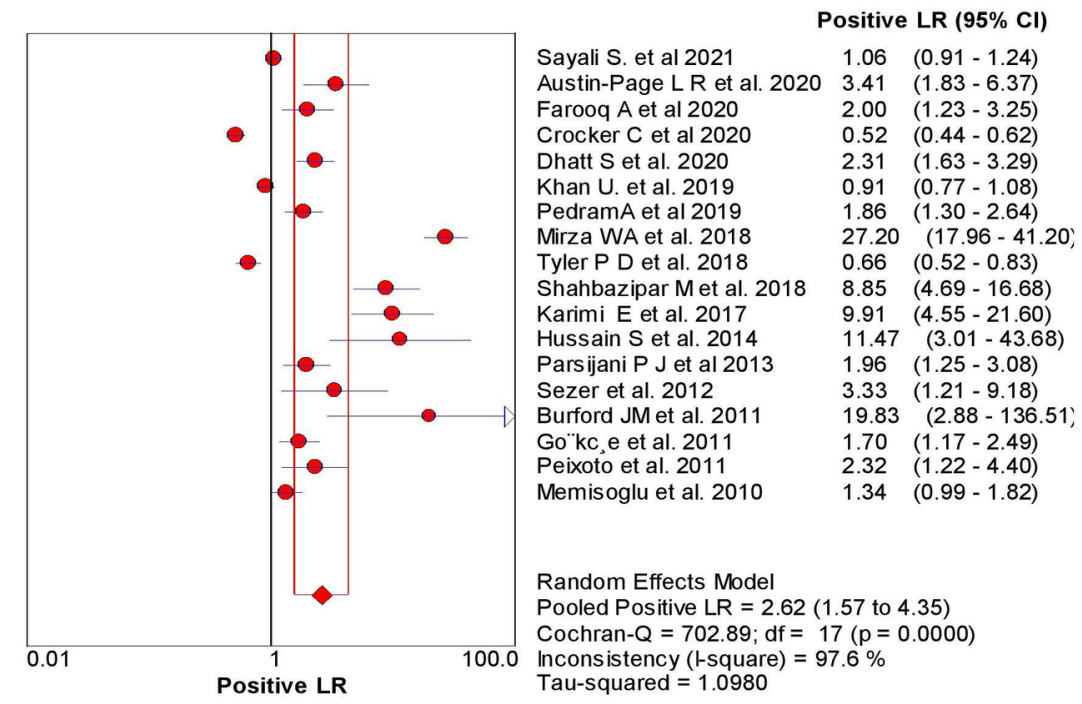

Figure 5: Negative Likelihood ratio of ultrasound in acute appendicitis cases

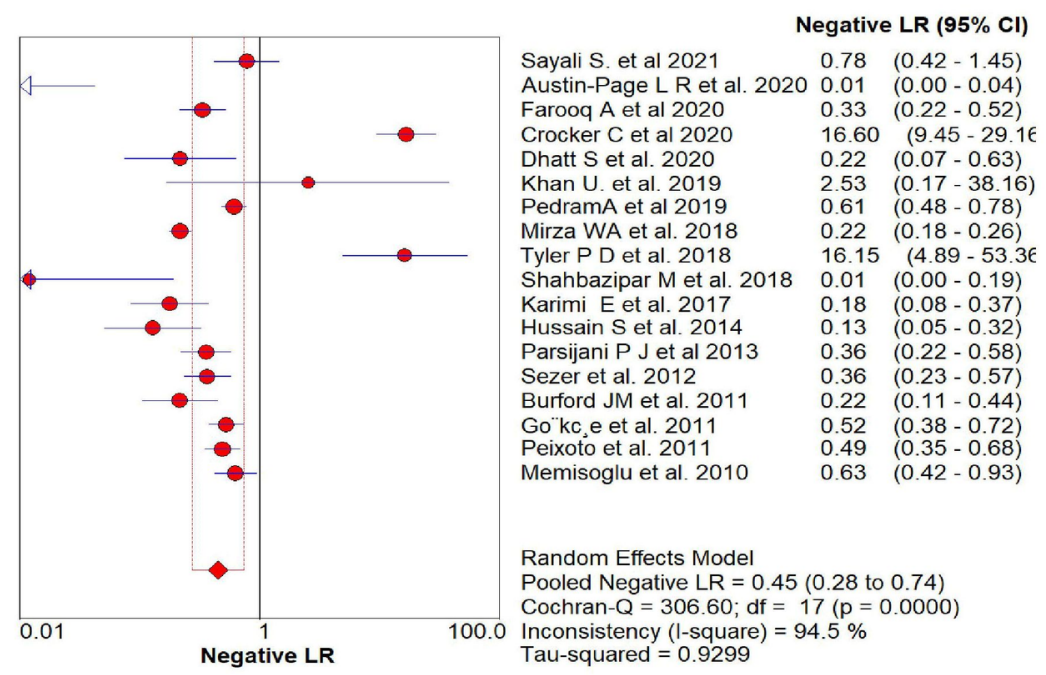


Figure 6: SROC curve of ultrasound in acute appendicitis cases

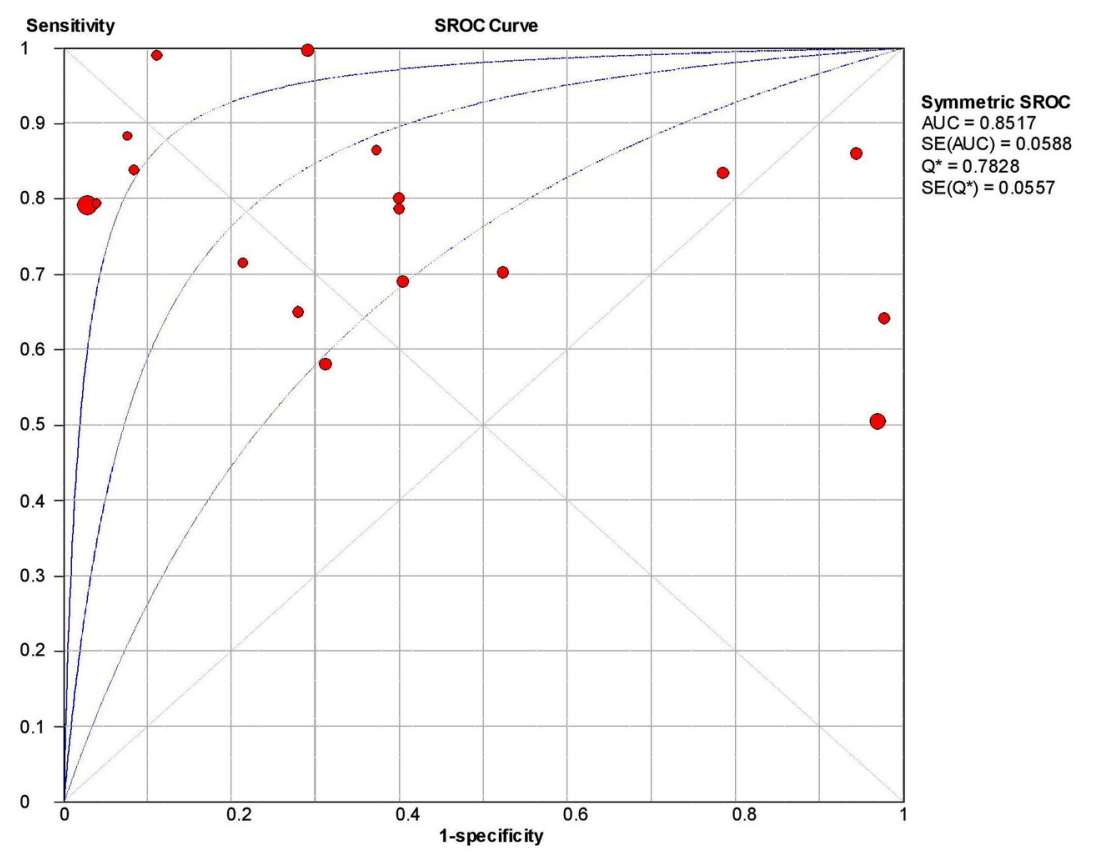

Figure 7: Diagnostic OR of ultrasound in acute appendicitis cases

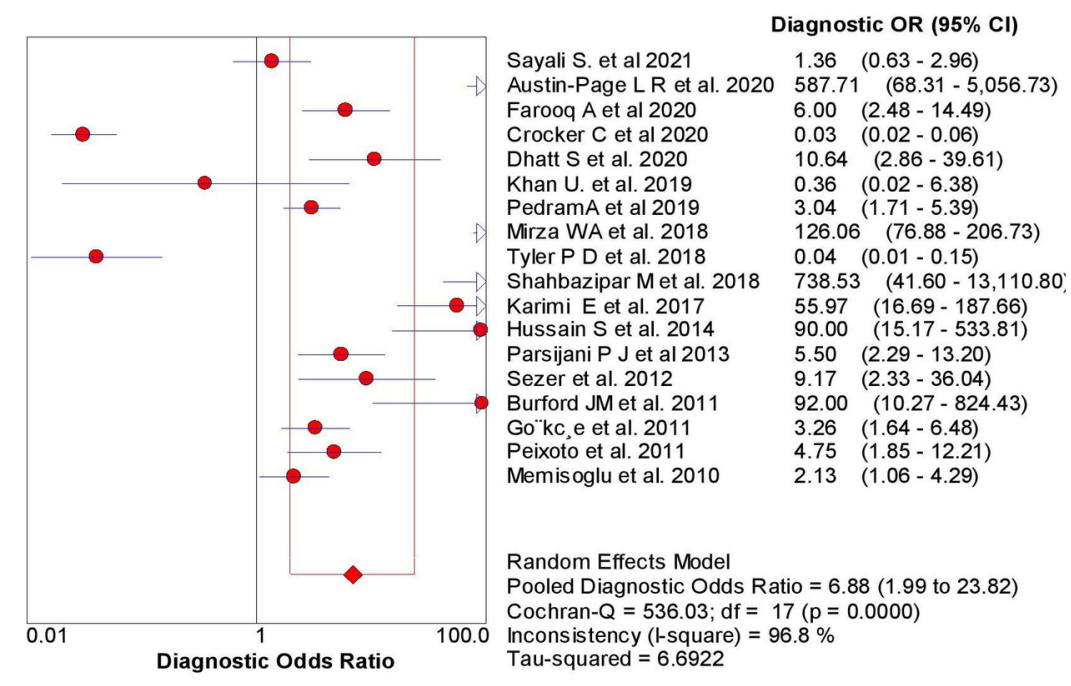




\section{Hosted file}

Table.docx available at https://authorea.com/users/415152/articles/523051-abdominalultrasound-and-its-diagnostic-accuracy-in-diagnosing-acute-appendicitis-a-meta-analysis 